\title{
Close similarity of the electronic structure and electron correlation in gas-phase and solid $\mathbf{C}_{60}$
}

\author{
S. Krummacher, M. Biermann, M. Neeb, A. Liebsch, and W. Eberhardt \\ Institut für Festkörperforschung der Forschungszentrum Jülich GmbH, P.O. Box 1913, D-52425 Jülich, Germany
}

(Received 8 March 1993)

\begin{abstract}
We present a study of the electronic structure and electron correlation effects in gas-phase and solid $\mathrm{C}_{60}$ clusters. Direct in situ comparison of $\mathrm{C} 1 s$ absorption spectra taken in the gas phase with those from solid $\mathrm{C}_{60}$ reveals a close similarity. This is also true for the shake-up satellites in the $\mathrm{C} 1 s \mathrm{x}$-rayphotoelectron spectrum. The main conclusions are that solid-state interactions play only a minor role in this new material, and electron correlation effects are predominantly intramolecular.
\end{abstract}

\section{INTRODUCTION}

Fullerite (solid $\mathrm{C}_{60}$ ) is the first pure solid cluster material obtainable in macroscopic quantities. ${ }^{1}$ Its properties differ remarkably from those of the ordinary solid carbon species, graphite or diamond. Accordingly, fullerite has attracted a lot of interest in the physics, chemistry, and materials science communities. This interest was additionally fueled when it was discovered that doping with alkali metals leads to superconductivity at fairly high temperatures $(30 \mathrm{~K})$. In a recent study it was proposed that these properties are strongly influenced by electron correlation effects in analogy to the high- $T_{c}$ cuprates. $^{2}$

One of the missing links in the studies so far is a detailed comparison of the electronic structure of a single $\mathrm{C}_{60}$ "molecule" or cluster with that of the solid. The same question arises with respect to the correlation effects. This prompted our investigation of the electronic properties of gas-phase $\mathrm{C}_{60}$ using electron yield and photoemission spectroscopy with synchrotron radiation.

We will present $\mathrm{C} 1 s$ photoabsorption spectra in order to investigate changes in the transitions from a $\mathrm{C} 1 s$ core level into the unoccupied valence orbitals. These orbitals should show the effect of band formation and dispersion in the solid. Moreover, high-resolution core-level photoemission will show the effects of the solid-state interaction through changes of the collective plasmon excitations. Additionally, the hole-hole Coulomb interaction $U$ will be determined from the binding energies of the core level, the valence orbitals, and the onset of the Auger electron emission.

\section{EXPERIMENTAL DETAILS}

Fullerite powder usually containing about $90 \% \quad \mathrm{C}_{60}$ was evaporated from a Knudsen furnace at roughly 960 $\mathbf{K}$. Only the powder used for the photoabsorption measurements had been purified by a liquid chromatography to reduce the contribution of higher fullerenes below the detection limit. At a distance of about $2 \mathrm{~mm}$ from the $0.5-\mathrm{mm}$-diameter nozzle the vapor beam was crossed at right angles by the synchrotron-radiation beam from the X1B soft-X-ray undulator beamline at the National Synchrotron Light Source (NSLS), Brookhaven, which is de- scribed in detail elsewhere. ${ }^{3,4}$ The photoelectrons were energy analyzed in a commercial cylindrical-mirroranalyzer-type electron spectrometer whose axis was oriented perpendicular to both the light and vapor beam. The $\mathrm{C}_{60}$ vapor was collected on a stainless-steel plate which could be moved into the interaction region after termination of the gas-phase measurements. This guaranteed an in situ comparison of gas-phase and solid spectra using the same energy calibration. Absolute energy calibration for the photon energy was obtained using the $1 s \rightarrow \pi^{*}$ transitions of gas phase $\mathrm{CO}$ and $\mathrm{CO}_{2}$ (Ref. 5) taken immediately before and after the $\mathrm{C}_{60}$ studies. Similarly, the kinetic-energy scale of the electron spectrometer was calibrated against the $K-L L$ Auger transition of $\mathrm{O}_{2} \cdot{ }^{6}$

\section{C $1 s$ TRANSITIONS INTO UNOCCUPIED STATES}

The upper panel of Fig. 1 shows a comparison of the onset of the $\mathrm{C} 1 \mathrm{~s}$ excitations into unoccupied electronic states in gas phase and solid $\mathrm{C}_{60}$. These spectra were obtained by measuring the secondary electron yield at kinetic energies between 2 and $10 \mathrm{eV}$ for the solid species, and the Auger electron yield at $250 \mathrm{eV}$ for the gas-phase species. They were normalized using the signal from a semiconductor photodiode located behind the interaction region in the direct photon beam in order to eliminate the variations of the incident photon flux in the region of the $C K$ edge. The band pass of the monochromator was set to $0.06 \mathrm{eV}$ for the gas-phase measurements, and $0.03 \mathrm{eV}$ for the measurements on the deposited film.

While for the gas phase no core absorption spectrum has been reported previously, several spectra already exist in the literature for the solid case, obtained either by photoabsorption ${ }^{7,8}$ or by electron-energy-loss spectroscopy. ${ }^{9,10}$ Our spectrum of solid $\mathrm{C}_{60}$ agrees well with those obtained with purified $\mathrm{C}_{60} \cdot{ }^{8-10}$ The peak observed at $287.3 \mathrm{eV}$ is due to the $1 s \rightarrow \pi^{*}$ transition in CO traces left in the chamber from the calibration measurement.

We do not want to discuss the difference in the slope of the continuous part of the two spectra, since this may be partly due to additional contributions in the solid-state spectrum caused by the presence of other carbon species in or on the steel plate. In order to emphasize the 
differences in the discrete excitations of $\mathrm{C}_{60}$, this continuous part of the cross section has been subtracted as a two segment linear "background" in the lower panel. The remaining differences are very subtle: The energy positions of all four features are identical within the accuracy of the experiment, but the amplitudes of peaks $B$ and $C$ in the solid spectrum relative to peak $A$ are reduced by $26 \%$ and $18 \%$, respectively. Also, peaks $A$ and $C$ in the gasphase spectrum show a clear broadening on the lowenergy side compared to the solid-state spectrum. Intuitively, one might have expected the solid-state features to be broadened due to band dispersion effects. However, here the solid spectrum has been taken at room temperature, while the gas-phase spectrum was taken at $960 \mathrm{~K}$. Therefore, the population of higher vibrational levels in the ground state can indeed lead to some broadening on the low-energy side of the peak. The full width at half maximum of the solid peak, on the other hand, is only 0.3 $\mathrm{eV}$, substantially lower than the theoretical dispersion of $\sim 0.5 \mathrm{eV}$ calculated by Saito and Oshiyama. ${ }^{11}$ Thus, we conclude that solid-state band dispersion does not play a major role in these spectra. In particular, no additional broadening and/or peak shifts are introduced in the

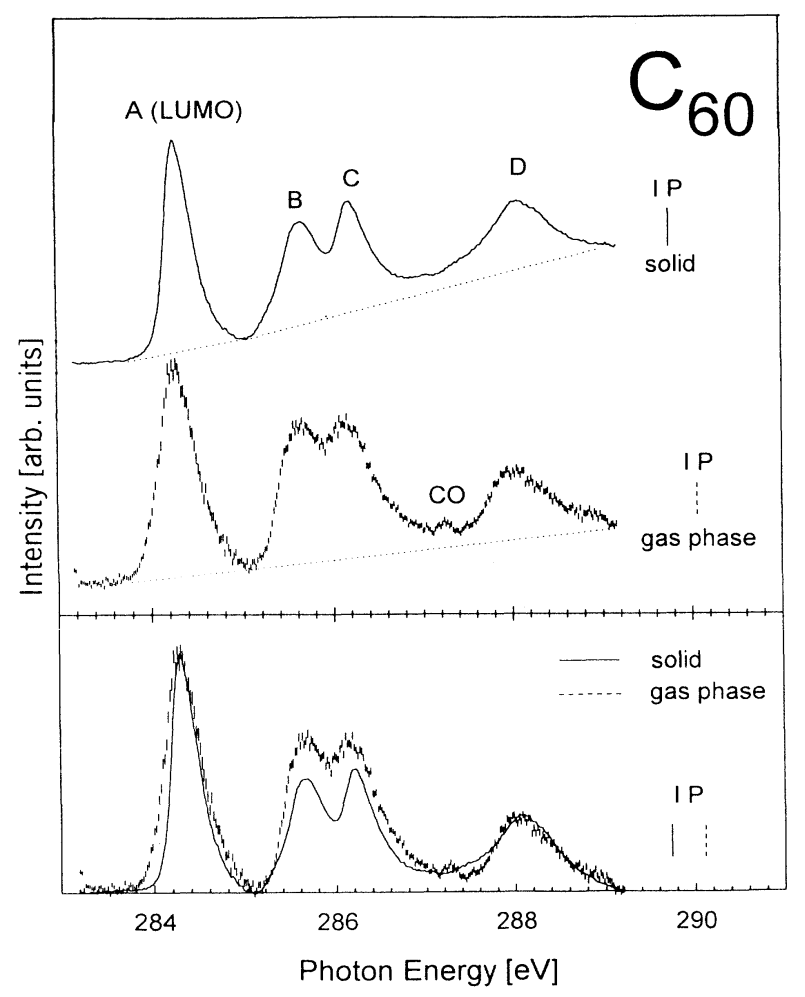

FIG. 1. Upper panel: C $1 s$ near-edge absorption spectra of gas-phase (error bars) and solid $\mathrm{C}_{60}$ (solid line). Lower panel: The same spectra after subtraction of the continuous part indicated by the dashed lines in the upper panel. The vertical bars at 290.1 and $289.75 \mathrm{eV}$ indicate the $\mathrm{C} 1 \mathrm{~s}$ ionization potential referenced to the vacuum level. The value for the solid has been obtained from the value of $282.9 \mathrm{eV}$ with respect to the HOMO reported by Weaver et al. (Ref. 14) and the value of $6.85 \mathrm{eV}$ for the separation between the HOMO and the vacuum level reported by Lichtenberger et al. (Ref. 16). solid-state spectra due to the electron final state. Even if all the observed states have a strong excitonic character, the peak energies should exhibit shifts due to the dispersion in the solid phase. This is due to the fact that the exciton splits off from the bottom of the band, whereas the gas-phase value of the transition should reflect the center of gravity of the bands.

\section{LOCALIZATION EFFECTS ON THE EXCITON BINDING ENERGY}

We can obtain information on the excitonic character of the first core-hole excited state ( $A$ in Fig. 1 ) by comparing the observed transition energy with the difference between the C $1 s$ ionization potential (IP) and the electron affinity of the lowest unoccupied molecular orbital (LUMO). For the gas phase, we have determined the IP to be $290.1 \mathrm{eV}$ by calibrating the photoelectron peak from the $\mathrm{C} 1 s$ core level in the X-ray-photoelectron spectrum (XPS) spectrum of $\mathrm{C}_{60}$ against that of $\mathrm{CO}$ reported in the literature. ${ }^{12}$ The vertical binding energy for an additional electron in the LUMO (without core or valence hole) can be obtained from the electron detachment spectrum reported by Haufler et al. ${ }^{13}$ Both energies are referred to the vacuum level. Combined with the observed transition energy of $284.3 \mathrm{eV}$, this yields an excitonic shift of $3.1 \mathrm{eV}$ caused by the Coulomb interaction between the core hole and the excited electron in the LUMO orbital. For the solid we have to use a different scheme to estimate the excitonic shift, since different types of measurements have been performed. Here we can combine the energy separation between the $\mathrm{C} 1 s$ and highest occupied molecular orbital (HOMO) measured in photoemission ${ }^{14}$ with the HOMO-LUMO separation of 3.5 (Ref. 2) or $3.7 \mathrm{eV}$ (Ref. 15) obtained from a comparison of direct and inverse photoemission data. Note that electron detachment and inverse photoemission probe exactly the same configuration (one additional electron in the LUMO and no core hole), the former as initial state, the latter as final state. We then arrive at an expected transition energy of 286.4 or $286.6 \mathrm{eV}$ for the $\mathrm{C} 1 \mathrm{~s}$ LUMO transition, compared to a measured value of $284.3 \mathrm{eV}$, yielding an excitonic shift of only 2.1 to $2.3 \mathrm{eV}$ for the solid. While the estimate for the solid hinges on the accuracy of the relative energy calibration between the direct and inverse photoemission measurements, the reduction in the excitonic shift of about $1 \mathrm{eV}$ compared to the isolated molecule could reflect a change in the localization of the excited electron. In a semiclassical description, such a reduction in the binding energy of the exciton corresponds to a proportional increase in the exciton radius. Since the core hole is localized at one atom, the radius actually reflects the localization of the bound electron.

\section{CHANGES IN THE HOLE-HOLE COULOMB REPULSION DUE TO LOCALIZATION}

The difference in the degree of (de)localization also leads to differences in the hole-hole Coulomb interaction energy $U$ of the valence electrons, which can be observed in the Auger decay of the core hole. Figure 2 shows the high-energy part of the Auger spectra of gas-phase and 


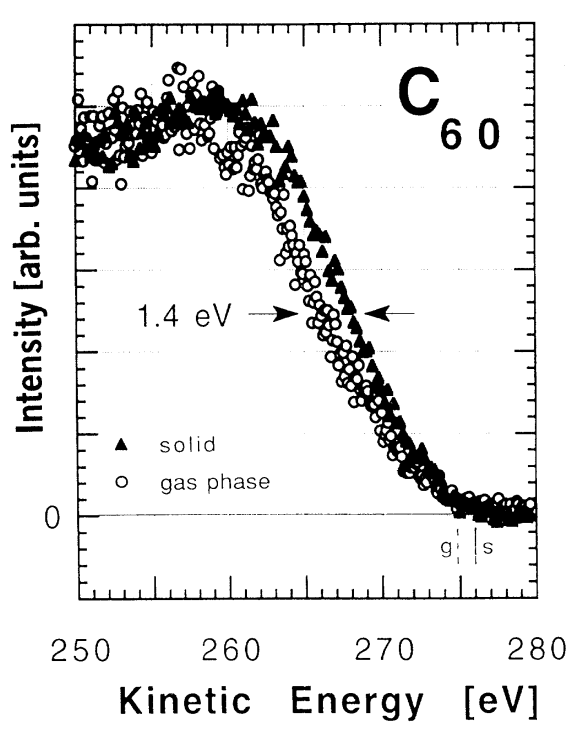

FIG. 2. $K-L L$ Auger spectra of gas-phase (open circles) and solid (solid triangles) $\mathrm{C}_{60}$ taken at $340-$ and $300-\mathrm{eV}$ photon energy, respectively. In the case of the solid spectrum, a smooth background due to secondary electrons has already been removed. The vertical bars indicate the maximum expected Auger energy for the gas phase (dashed line) and the solid (solid line) if the Coulomb energy $U$ is neglected. The kinetic energy is referenced to the vacuum level.

solid $\mathrm{C}_{60}$ after complete removal of the $\mathrm{C} 1 \mathrm{~s}$ electron by 340 or $300 \mathrm{eV}$ photons, respectively. In the case of the solid a smooth background due to secondary electrons has already been subtracted. The kinetic-energy scale is referenced to the vacuum level and has been calibrated against the $K-L L$ Auger transition of $\mathrm{O}_{2}{ }^{6}$ The Auger electrons with the maximum kinetic energy must originate from the process involving two electrons in the HOMO, so that

$$
E_{k}^{\max }(\text { Auger })=E_{B}(1 s)-2 \times E_{B}(\mathrm{HOMO})-U,
$$

where $E_{k}{ }^{\max }$ (Auger) is the maximum observed Auger energy, and $E_{B}(1 s)$ and $E_{B}(\mathrm{HOMO})$ are the binding energies of the $1 s$ core hole and valence level, respectively, both referenced to the vacuum level. $U$ represents the Coulomb interaction energy between the two valence holes. Using the aforementioned values for the binding energies and neglecting $U$ one would thus expect the onset of the Auger spectrum of the solid at $276.1 \mathrm{eV}$. Similarly, for the gas phase this onset would be expected at $274.9 \mathrm{eV}$, using the value of $7.61 \mathrm{eV}$ for the HOMO binding energy ${ }^{16}$ and again neglecting $U$. These two energies are marked by the vertical bars at the bottom of Fig. 2 . The value of $U$ can, in principle, be read directly from the difference between these values and the true onset of the spectra. While this onset is difficult to determine quantitatively, the spectra clearly show that $U$ has to be quite small.

The relative shift between the Auger spectra of gasphase and solid $\mathrm{C}_{60}$, however, can be determined with much higher accuracy. The kinetic energy at the steepest slope of the solid Auger spectrum is about $1.4 \mathrm{eV}$ higher than that of the gas-phase spectrum. Taking the differences in the binding energies into account results in a gas-phase correlation energy that is only $0.23 \mathrm{eV}$ larger than in the solid. This difference is much smaller than earlier estimates $(3.4 \mathrm{eV}$ for the gas phase and $1.6 \mathrm{eV}$ for the solid $^{2}$ ). Moreover, if the simple polarization model presented in Ref. 2 is applicable, then our results would imply that $U$ in the solid is also only a few tenths of an $\mathrm{eV}$. On the other hand, at the present state we cannot fully exclude that our results are reduced by a small $C_{70}$ contribution, since only the $\mathrm{x}$-ray-absorption near-edge structure measurements were performed with purified material. However, this effect should be rather small.

\section{CORRELATION EFFECTS IN THE CORE-LEVEL PHOTOEMISSION}

Electron correlation effects are also reflected in the satellite structure observed in core-level photoemission. Figure 3 shows the gas-phase $\mathrm{C}_{60} \mathrm{C} 1 \mathrm{~s}$ photoelectron spectrum after ionization with 390-eV photons compared to the corresponding spectrum obtained by Weaver et al. ${ }^{14}$ for solid $\mathrm{C}_{60}$ using $\mathrm{Al} K \alpha$ radiation. In both cases, the core ionization is accompanied by pronounced and extensive satellite structure. Roughly, this structure can be divided into two regions, namely a sequence of discrete shake-up peaks extending from the $\mathrm{C} 1 \mathrm{~s}$ main line to roughly $16 \mathrm{eV}$, and a broad feature between about 16 and $50 \mathrm{eV}$.

\section{Collective excitations}

We shall discuss these two regions separately, beginning with the "plasmon" region between 16 and $50 \mathrm{eV}$. This region is dominated by collective excitations due to the Coulomb interaction between the core hole and the 240 valence electrons in the cluster. In our $\mathrm{C} 1 s$ photoemission spectrum of gas-phase $\mathrm{C}_{60}$ they give rise to the broad structure peaked at about $34 \mathrm{eV}$. In the solid, a structure with approximately the same width is observed

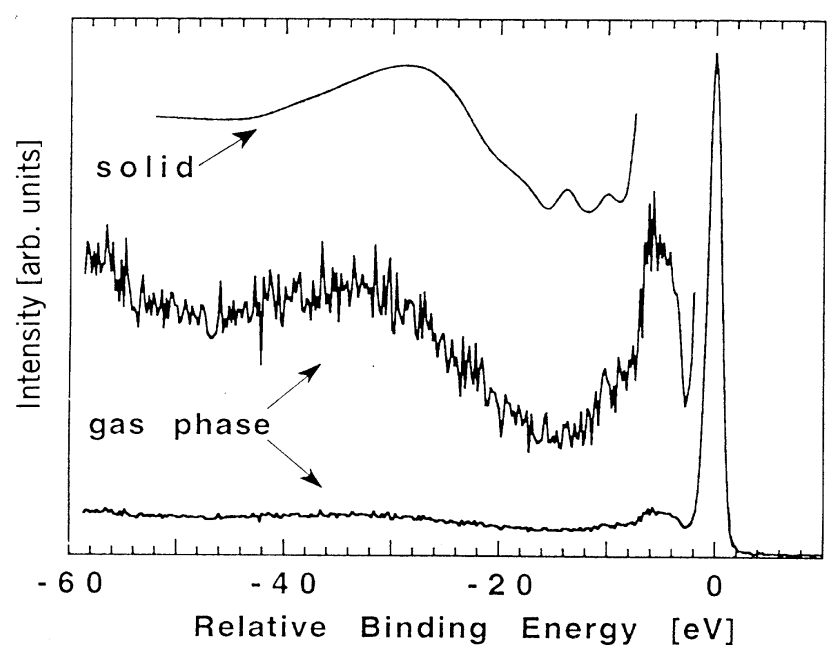

FIG. 3. C $1 s$ photoelectron spectra of gas-phase $\mathrm{C}_{60}$ at 390$\mathrm{eV}$ photon energy and of solid $\mathrm{C}_{60}$ at $1487 \mathrm{eV}$. The solid spectrum is from Ref. 13. 
at a somewhat lower energy of about $28 \mathrm{eV} .^{14}$ Similarly, in optical-absorption spectra of gas-phase $\mathbf{C}_{60}$ a giant plasmon has been observed by Hertel et al. ${ }^{17}$ at a photon energy of $19 \mathrm{eV}$ with a total width of about $10 \mathrm{eV}$, while in the electron-energy-loss spectrum of solid $\mathrm{C}_{60}$ this structure appears again at about $28 \mathrm{eV} .^{9,10}$

In order to understand these spectroscopic observations we have performed model calculations for the $\mathrm{C}_{60}$ molecular polarizabilities and for the solid $\mathrm{C}_{60}$ loss function. We represent the main electronic transition contributing to the $\sigma$ plasmon by a Lorentz oscillator of the form

$$
\varepsilon_{L}(\omega)=1+\frac{4 \pi n}{\left(\omega_{0}^{2}-\omega^{2}-i \omega \Gamma\right)}
$$

where $\omega_{0}$ is the frequency characteristic of the $\sigma$ excitations, $\Gamma$ accounts for the width of the absorption line, and $n=240 / V$ is the molecular electronic density. Because of the shell structure of the $\mathrm{C}_{60}$ molecule, the electrons are assumed to be located in the region $\rho \leq r \leq R$, i.e., $V=4 \pi\left(R^{3}-\rho^{3}\right) / 3$. $R$ and $\rho$ are taken to be symmetrical about the radial position of the $C$ nuclei, $3.5 \AA$. The same model was recently used by Lambin, Lucas, and Vigneron ${ }^{18}$ to study the cohesive properties of solid $\mathrm{C}_{60}$. The imaginary parts of the normalized molecular multipole polarizabilities $\alpha_{l}(\omega) /\left(l R^{3}\right)$ for this model are shown in Fig. 4. The parameters in this example are $\Gamma=7 \mathrm{eV}, \rho=3 \AA$, and $R=4 \AA$. As a result of the empty interior, the usual particle Mie resonance and all higher plasma modes are split. The frequency of the main line of the dipole polarizability at $19 \mathrm{eV}$ agrees with the mean position of the absorption spectrum observed by Hertel et al. ${ }^{17}$ On the other hand, since the satellites of the $\mathrm{C} 1 \mathrm{~s}$ core-level photoelectron spectra shown in Fig. 3 do not need to obey the $l=1$ selection rule, they should consist of a superposition of all multipole polarizabilities. It is therefore not surprising that the centroid of the $\mathrm{C} 1 s$ core-level satellite is shifted to higher energies, and that the width of the satellite feature is significantly larger than that of the photoabsorption spectrum.

Also shown in Fig. 4 is the solid $\mathrm{C}_{60}$ loss function which we derive from the Clausius-Mosotti relation

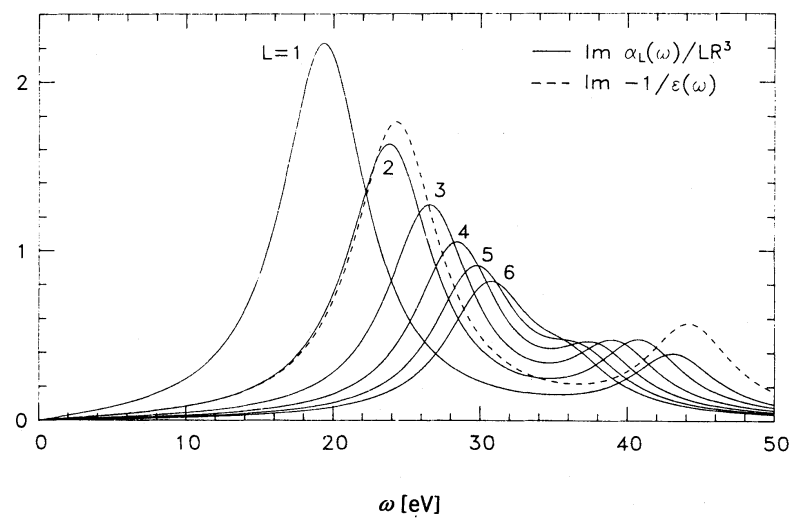

FIG. 4. Imaginary parts of normalized molecular polarizabilities $\alpha_{l}(\omega) /\left(l R^{3}\right)$ of $\mathrm{C}_{60}$ for $l=1, \ldots, 6$ (solid lines) and loss function $\operatorname{Im}[-1 / \varepsilon(\omega)]$ of solid $\mathbf{C}_{60}$ (dotted line).

$$
\frac{\varepsilon(\omega)-1}{\varepsilon(\omega)+2}=\frac{4 \pi}{3} \times \frac{4}{a^{3}} \times \alpha_{l=1}(\omega),
$$

where $a=14.2 \AA$ is the lattice constant of the fcc lattice for solid $\mathrm{C}_{60}$. The dipole-dipole interaction between the molecules forming the solid is seen to shift the molecular Mie plasmon from 19 to about $25 \mathrm{eV}$. As shown in Ref. 18 , including the higher multipole polarizabilities can increase this frequency somewhat and can also affect the shape of the solid plasmon spectrum. This result agrees well with the electron-energy-loss spectra recently observed for solid $\mathrm{C}_{60} \cdot{ }^{9,10}$ This plasmon ("polarization wave") excitation presumably is also the main mechanism contributing to the $1 s$ core-level satellite in solid $\mathrm{C}_{60}$. Nevertheless, as a result of matrix element effects, the relative weights of the multipole polarizabilities contributing to the satellite might not be precisely the same as in the electron-energy-loss spectrum. At the same time the core-level satellite in solid $\mathrm{C}_{60}$ should also differ from the corresponding gas-phase spectrum because of the excitation of the polarization wave in the solid. This is evident in Fig. 3 which shows that the mean binding energies of these satellites differ by about $6.5 \mathrm{eV}$.

The above model for the dynamic response of the valence electrons of molecular and solid $\mathbf{C}_{60}$ helps to qualitatively understand the differences between the various spectroscopic observations. We point out, however, that the calculated spectra depend strongly on the parameters used and that the matrix elements that are relevant for each particular case have an important influence on the spectral distributions. Moreover, both the valence photoabsorption spectra ${ }^{17}$ and the electron-energy-loss spectra ${ }^{9,10}$ show prominent fine structure in the molecular and solid plasmon peaks. These features can only be accounted for within more refined response calculations.

\section{Shake-up structures}

Let us now turn to the region of the discrete satellites between the C $1 s$ line and about 16-eV "loss energy." Figure 5 shows the spectrum on an expanded energy scale, again compared to the corresponding spectrum for the solid obtained by Weaver et al. ${ }^{14}$ using Al $K \alpha$ radiation. We observe several distinct structures in this energy

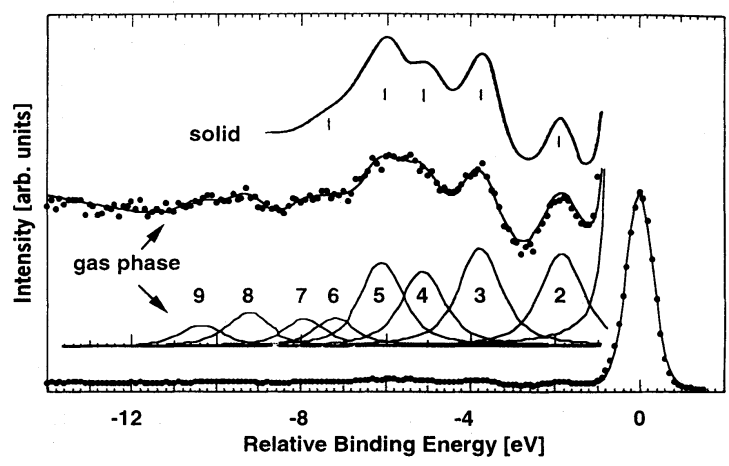

FIG. 5. C $1 s$ photoelectron spectra of gas-phase and solid $\mathrm{C}_{60}$ from Fig. 3 on an expanded energy scale together with a leastsquares fit. 
TABLE I. Shake-up components of the $C 1 s$ spectrum of $\mathrm{C}_{60}$ of Fig. 5 based on a least-squares fit.

\begin{tabular}{|c|c|c|c|c|}
\hline \multirow[b]{2}{*}{ Peak } & \multirow{2}{*}{$\begin{array}{l}\text { Shake-up } \\
\text { transition }\end{array}$} & \multicolumn{2}{|c|}{$\begin{array}{c}\text { Excitation energy } \\
(\mathrm{eV})\end{array}$} & \multirow{2}{*}{$\begin{array}{c}\text { Intensity } \\
(\%) \\
\text { Gas phase }\end{array}$} \\
\hline & & Gas phase & Solid (Ref. 14) & \\
\hline 1 & C $1 s$ main line & 0.00 & 0.00 & 100 \\
\hline 2 & $\begin{aligned} h_{u} & \rightarrow t_{1 u} \\
(\text { HOMO } & \rightarrow \text { LUMO) }\end{aligned}$ & -1.9 & -1.8 & 6.1 \\
\hline 3 & $\begin{array}{l}h_{u} \rightarrow t_{2 u}, h_{g} \\
(\mathrm{HOMO} \rightarrow C)\end{array}$ & -3.8 & -3.7 & 6.5 \\
\hline 4 & $\begin{array}{c}g_{g}, h_{g} \rightarrow t_{2 u}, h_{g} \\
(\mathrm{HOMO}-1 \rightarrow C)\end{array}$ & -5.1 & -5.0 & 5.0 \\
\hline 5 & $\begin{array}{c}h_{u} \rightarrow h_{u}, g_{g}, g_{u}, t_{g} \\
\text { (HOMO } \rightarrow D \\
\text { and } \pi \text { plasmon) }\end{array}$ & -6.1 & -6.0 & 5.6 \\
\hline 6 & $\begin{array}{l}g_{g}, h_{g} \rightarrow h_{u}, g_{g}, g_{u}, t_{g} \\
(\mathrm{HOMO}-1 \rightarrow D)\end{array}$ & -7.2 & -7.2 & 1.9 \\
\hline 7 & & -7.9 & & 1.8 \\
\hline 8 & & -9.2 & & 2.8 \\
\hline 9 & & -10.4 & & 1.4 \\
\hline
\end{tabular}

region, which can be represented by at least eight components as indicated in Fig. 5. More details of this leastsquares fit are given in Table I. For this fit we used Voigt profiles of a constant total width of $0.7 \mathrm{eV}$ for the main line and $1.0 \mathrm{eV}$ for each of the satellite components. The main conclusion from this comparison is that the shakeup features for gas-phase and solid $\mathrm{C}_{60}$ are essentially identical.

For a few of these shake-up satellites a tentative assignment can be given. In particular, peak 2 is assigned to a shake-up transition from the HOMO to the LUMO accompanying the $\mathrm{C} 1 s$ ionization. Again, the observed energy of $1.9 \mathrm{eV}$ does not reflect the ground-state HOMOLUMO gap of $3.5 \mathrm{eV}$ but rather the transition energy modified by electron correlation. The energy separation of peaks 3 and 5 relative to peak 2 in the shake-up spectrum corresponds to the energy separation of peaks $C$ and $D$ from peak $A$ observed in the absorption spectrum. Accordingly, these features are assigned to shake-up transitions from the HOMO to the corresponding higher unoccupied states. The energy separation between peaks 3 and 4 reflects the energy difference between the HOMO and the next lower valence orbital, ${ }^{16}$ so that the tentative assignment of this peak is a shake-up transition from the HOMO-1 to $C$. Similarly, peak 6 can be assigned to a HOMO- $1 \rightarrow D$ transition.

In addition to that peak 5 might also contain a contribution due to a collective excitation involving only $\pi$ electrons. Evidence for such a low-energy plasmon at 6.3-eV excitation energy has recently been obtained from the energy dependence of high-resolution electronenergy-loss spectroscopy measurements of solid $\mathrm{C}_{60} \cdot{ }^{9}$ The absence of dipole-dipole interaction between neighboring molecules in the gas phase could lead to modifications of this transition in the isolated molecule and thus explain the differences observed by us between the gas phase and solid spectrum in this region.

\section{CONCLUSION}

Our in situ comparison between the absorption nearedge structure as well as the shake-up satellite structure of gas phase and solid $\mathrm{C}_{60}$ show that band-structure effects do not play any significant role in the unoccupied states of this new material. The increased linewidth of the $\mathrm{C} 1 s$ absorption spectrum in the gas phase is attributed to the population of excited vibronic states in the $\mathrm{C}_{60}$ beam prior to the excitation. Nevertheless, the different degree of localization does lead to changes in the Coulomb energy $U$ between two valence holes as well as in the exciton binding energy between a core hole and an electron in the LUMO orbital. The observed hole-hole interaction energy is substantially smaller than previously determined values, and the difference between the solid and the gas-phase for $U$ is only about $0.2 \mathrm{eV}$. The differences in the collective plasmon excitation are due to different symmetry selection rules and the contribution of different multipole moments. In general, all electron correlation effects observed are mainly a property of the individual $\mathrm{C}_{60}$ cluster, so that it is unlikely that they reflect upon the mechanism of macroscopic conductivity or even superconductivity of the solid.

\section{ACKNOWLEDGMENTS}

It is a pleasure to acknowledge the skillful help of F. P. Johnen, F. Loeb, and the staff of the National Synchrotron Light Source. Fruitful discussions with B. Kessler, T. Schober, and F. Hudson are also gratefully acknowledged. The experiments were carried out on beamline $\mathrm{X} 1 \mathrm{~B}$ at the NSLS Brookhaven, which is supported by the DOE, Office of Basic Energy Sciences. 
${ }^{1}$ W. Krätschmer, L. D. Lamb, K. Fostiropoulos, and D. R. Huffman, Nature 347, 354 (1990).

${ }^{2}$ R. W. Lof, M. A. van Veenendaal, B. Koopmans, H. T. Jonkman, and G. A. Sawatzky, Phys. Rev. Lett. 68, 3924 (1992).

${ }^{3}$ W. Eberhardt, K. J. Randall, J. Feldhaus, A. M. Bradshaw, R. F. Garrett, and M. L. Knotek, Phys. Scr. 41, 745 (1990).

${ }^{4}$ K. J. Randall, J. Feldhaus, W. Erlebach, A. Bradshaw, W. Eberhardt, Z. Xu, Y. Ma, and P. D. Johnson, Rev. Sci. Instrum. 63, 1367 (1992).

${ }^{5}$ G. R. Wight and C. E. Brion, J. Electron. Spectrosc. 3, 191 (1974); R. P. Hitchcock and C. E. Brion, ibid. 18, 1 (1980).

${ }^{6}$ W. E. Moddeman, T. A. Carlson, M. O. Krause, B. P. Pullen, W. E. Bull, and G. K. Schweitzer, J. Chem. Phys. 55, 2317 (1971).

${ }^{7}$ S. L. Molodtsov, A. Gutierrez, M. Domke, and G. Kaindl, Europhys. Lett. 19, 369 (1992).

${ }^{8}$ L. J. Terminello, D. K. Shuh, F. J. Himpsel, D. A. LapianoSmith, J. Stoehr, D. S. Bethune, and G. Meijer, Chem. Phys. Lett. 182, 491 (1991).

${ }^{9}$ G. Gensterblum, J. J. Pireaux, P. A. Thiry, R. Caudano, J. P. Vigneron, Ph. Lambin, A. A. Lucas, and W. Krätschmer, Phys. Rev. Lett. 67, 2171 (1991).

${ }^{10}$ E. Sohmen, J. Fink, and W. Kraetschmer, Z. Phys. B 86, 87
(1992).

${ }^{11}$ S. Saito and A. Oshiyama, Phys. Rev. Lett. 66, 2637 (1991).

${ }^{12}$ A. Hitchcock and C. Brion, J. Electron. Spectrosc. 18, 1 (1981).

${ }^{13}$ R. E. Haufler, L. S. Wang, L. P. F. Chibante, C. Jin, J. Conceicao, Y. Chai, and R. E. Smalley, Chem. Phys. Lett. 179, 449 (1991).

${ }^{14}$ J. H. Weaver, J. L. Martins, T. Komeda, Y. Chen, T. R. Ohno, G. H. Kroll, N. Troullier, R. E. Haufler, and R. E. Smalley, Phys. Rev. Lett. 66, 1741 (1991).

${ }^{15}$ J. H. Weaver, J. Phys. Chem. Solids 53, 1433 (1992).

${ }^{16}$ D. L. Lichtenberger, M. E. Jatcko, K. W. Nebesny, C. D. Ray, D. R. Huffman, and L. D. Lamb, in Clusters and Cluster-Assembled Materials, edited by R. S. Averback, J. Bernholc, and D. L. Nelson, MRS Symposia Proceedings No. 206 (Materials Research Society, Pittsburgh, 1991), p. 673; D. L. Lichtenberger, K. W. Nesbesny, C. D. Ray, D. R. Huffman, and L. D. Lamb, Chem. Phys. Lett. 176, 203 (1991).

${ }^{17}$ I. V. Hertel, H. Steger, J. de Vries, B. Weisser, C. Menzel, B. Kamke, and W. Kamke, Phys. Rev. Lett. 68, 784 (1992).

${ }^{18}$ Ph. Lambin, A. A. Lucas, and J. P. Vigneron, Phys. Rev. B 46, 1794 (1992). 